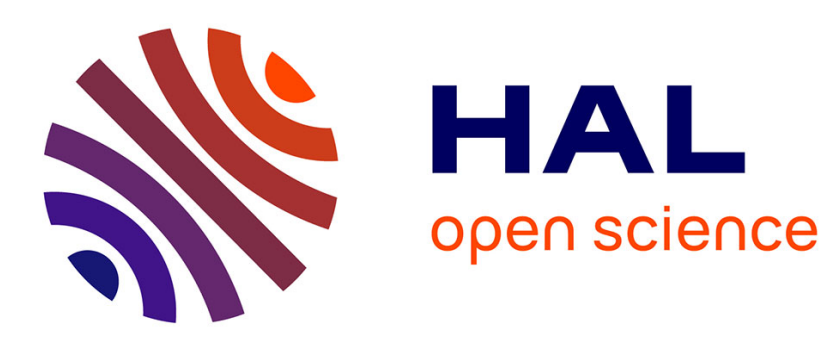

\title{
Aptitude à la coagulation du lait de vache: influence de l'alimentation: Etude réalisée dans 6 exploitations du Pays de Thônes (Haute-Savoie)
}

\author{
B. Martin, J.B. Coulon
}

\section{- To cite this version:}

B. Martin, J.B. Coulon. Aptitude à la coagulation du lait de vache: influence de l'alimentation: Etude réalisée dans 6 exploitations du Pays de Thônes (Haute-Savoie). Productions Animales, 1991, 4 (3), pp.209-217. hal-00895940

\author{
HAL Id: hal-00895940 \\ https://hal.science/hal-00895940
}

Submitted on 1 Jan 1991

HAL is a multi-disciplinary open access archive for the deposit and dissemination of scientific research documents, whether they are published or not. The documents may come from teaching and research institutions in France or abroad, or from public or private research centers.
L'archive ouverte pluridisciplinaire HAL, est destinée au dépôt et à la diffusion de documents scientifiques de niveau recherche, publiés ou non, émanant des établissements d'enseignement et de recherche français ou étrangers, des laboratoires publics ou privés. 
INRA Prod. Anim., 1991, 4 (3), $209-217$

\section{B. MARTIN, J.B. COULON*}

SUACI Montagne Alpes du Nord 1, rue du Château - 73000 Chambéry

* INRA Laboratoire de la Lactation et de l'Elevage des Ruminants Theix 63122 Saint-Genès-Champanelle
Aptitude à la coagulation du lait de vache : influence de l'alimentation

\author{
Etude réalisée dans \\ 6 exploitations du Pays \\ de Thônes (Haute-Savoie)
}

\section{L'évolution rapide des facteurs de production et des contraintes de ramassage et de transformation du lait a entraîné des modifications de sa qualité qui peuvent avoir des répercussions sur la quantité et la qualité des produits transformés. Ces modifications sont particulièrement importantes dans les régions où la majeure partie du lait est transformée en fromages, ce qui est notamment le cas des Alpes du Nord.}

\begin{abstract}
Dans la plupart des régions de montagne, la valorisation du lait est exclusivement fromagère et l'ensemble de l'agriculture repose sur la fabrication, dans de petites unités de production, de fromages à base de lait cru en Appellation d'Origine Contrôlée. Un cahier des charges très strict, qui garantit la qualité des produits, interdit les modifications artificielles de la composition du lait. Or, les laits de petits mélanges utilisés en production fromagère dans les Alpes du Nord présentent souvent une grande variabilité au cours de l'année. Ceci ne va pas sans poser des problèmes aux fromagers qui, du fait
\end{abstract}

\section{Résumé}

L'étude réalisée entre janvier et juillet 1989 dans six exploitations laitières réalisant du Reblochon fermier dans le Pays de Thônes (Haute-Savoie) a permis de décrire les variations des principaux paramètres de l'aptitude à la coagulation des laits individuels et de troupeau (temps de coagulation, vitesse de raffermissement, fermeté du gel) et de les relier aux caractéristiques quantitatives de l'alimentation hivernale des animaux (bilans énergétique, azoté et minéral). Ces mesures ont permis de montrer les particularités des conduites alimentaires des troupeaux de cette région où globalement, les apports alimentaires hivernaux ont été largement supérieurs aux besoins des animaux $(+1$ à $+25 \%)$. Les excédents se sont accompagnés de déséquilibres des apports azotés (excès de PDIE par rapport aux PDIN) et minéraux (excès de calcium par rapport au phosphore) et ont surtout été marqués (excédents énergétiques de l'ordre de $20 \%$ ) dans les exploitations caractérisées par des laits de qualité beaucoup plus variable au cours de l'année. A l'échelle des troupeaux $(n=6)$ comme à l'échelle de l'individu $(n=160)$, il n'a cependant pas été possible, dans la limite des données disponibles, de mettre en relation l'aptitude à la coagulation du lait avec les bilans alimentaires des animaux. L'étude des laits individuels a par contre permis de confirmer l'influence du stade physiologique des animaux et du $\mathrm{pH}$ initial du lait sur ses paramètres de coagulation. Les caractéristiques des laits des troupeaux ont été très liées à l'exploitation et d'autres facteurs, alimentaires, ou non pourraient être en cause pour expliquer les différences d'aptitude du lait à la coagulation. de l'automatisation croissante des chaînes de production, ont du mal à adapter leurs techniques de fabrication aux variations des caractéristiques du lait: composition, comportement vis à vis de la présure, caractéristiques rhéologiques du caillé...

Il est donc primordial de mieux connaître les causes des variations de l'aptitude fromagère des laits, en particulier dans les conditions spécifiques des Alpes du Nord (alimentation à base de foin, races locales..), afin, pour les éleveurs d'agir sur les facteurs de variabilité et produire un lait de qualité la plus constante possible au cours de l'année, et pour les fromagers de mieux savoir gérer cette variabilité et reconnaître les situations à risque.

Un suivi en ferme réalisé en 1988 dans 41 exploitations du Pays de Thônes (Haute-Savoie) (Coulon et al 1988) a montré qu'il existait d'importantes variations de la composition chimique et de l'aptitude à la coagulation du lait (mesurée par le temps de coagulation) bien que l'on se situe dans une zone géographique limitée avec des animaux d'une seule race (Abondance), recevant tous une alimentation à base de foin. Ces variations semblaient en relation avec la maîtrise du système d'élevage et plus particulièrement de l'alimentation.

Compte-tenu de ces premiers résulats, l'objectif de cette étude a été d'observer et d'analyser, dans 6 exploitations faisant partie de l'enquête précédente, l'évolution des principaux paramètres de l'aptitude à la coagulation des laits et de les relier aux caractéristiques quantitatives de l'alimentation hivernale des animaux.

Etude réalisée dans le cadre du programme de Recherche-Développement des Alpes du Nord 


\section{1 / Origine et analyse des données}

Les 6 exploitations sont localisées en HauteSavoie dans le canton de Thônes et fabriquent du Reblochon fermier. Les vaches sont toutes de race Abondance et l'alimentation hivernale est à base de foin, acheté ou produit sur place. Trois des 6 exploitations faisaient partie du groupe dont le lait présentait de fortes variations du temps de coagulation au cours de l'hiver (groupe "variable»-V-), les trois autres faisaient partie du groupe d'exploitations dont le temps de coagulation du lait restait stable au cours de l'hiver (groupe « stable »-S-) (cf Coulon et al 1988).

\section{1 / Données recueillies}

\section{a / Alimentation}

Trois fois au cours de l'hiver 1989 (première quinzaine de janvier, dernière semaine de février-première semaine de mars, dernière quinzaine d'avril), les quantités ingérées ont été mesurées dans chaque exploitation (individuellement pour le concentré et à l'échelle du troupeau pour les fourrages). Chaque série de mesure comprenait trois jours consécutifs de pesée des quantités de foin (offert à volonté) et de concentré distribuées et refusées. Les mesures ont toujours été réalisées au moins 5 jours après un changement important de lot de fourrage.

Le deuxième jour de chaque série de pesées, un échantillon de chaque lot de fourrage distribué a été prélevé pour déterminer les teneurs en matière sèche (MS), matière organique (MO), cellulose brute (CB), matières azotées totales (MAT), et minéraux (phosphore et calcium) ainsi que la digestibilité de la matière organique (test pepsine-cellulase). Ces données ont permis de déterminer la valeur nutritive des aliments distribués (teneurs en UFL, PDIN, PDIE, $\mathrm{P}$ et $\mathrm{Ca}$ ) (tableau 1). Les fourrages distribués ont été caractérisés par leur richesse en PDIE (l'écart PDIE-PDIN a été en moyenne de $11,4 \mathrm{~g}$ PDI/UFL) et en calcium $(9,4 \mathrm{~g} / \mathrm{kg}$ MS). Les foins distribués ont généralement été de meilleure qualité dans les 3 exploitations du groupe $\mathrm{S}(+0,04 \mathrm{UFL} / \mathrm{kg} \mathrm{MS}$ et $+8 \mathrm{~g} \mathrm{PDI} / \mathrm{kg}$ MS).
Les bilans alimentaires (UFL, PDI, $\mathrm{P}$ et $\mathrm{Ca}$ ) ont été calculés pour chaque série de mesure en tenant compte des phénomènes d'interactions digestives et métaboliques (Vermorel et al 1987). Pour le fourrage, seules les quantités globalement ingérées par le troupeau étant connues, la consommation totale a été ramenée à une consommation moyenne égale pour tous les animaux présents. Ces bilans n'ont donc de signification stricte qu'à l'échelle du troupeau ; à l'échelle individuelle, ils renseignent cependant mieux que la seule connaissance des quantités de concentré ingérées.

\section{b / Production et composition du lait}

Sur les laits individuels, des mesures mensuelles ont été réalisées entre janvier et juillet 1989 : chaque série de mesure comprenait un prélèvement sur le lait de la traite du soir directement amené au laboratoire et conservé à $4^{\circ} \mathrm{C}$ pendant $15 \mathrm{~h}$ (sans conservateur) et un prélèvement sur celui de la traite du matin. Les taux butyreux et protéiques ont été déterminés séparément sur un échantillon de chaque traite (matin et soir). La numération cellulaire et le dosage du calcium ont été réalisés sur un échantillon moyen des deux traites. L'urée, le $\mathrm{pH}$, le degré d'acidité Dornic, le temps de coagulation et l'indice de coagulation (rapport entre le temps de coagulation de l'échantillon et le temps de coagulation d'un lait témoin reconstitué emprésuré avec la même solution de présure) ont été mesurés sur les laits du matin seulement, dès l'arrivée des échantillons au laboratoire ( 2 heures après la traite).

Sur les laits de mélange, deux fois par mois, entre janvier et juillet inclus, des échantillons des traites du matin et du soir ont été prélevés avant l'ensemencement, dans les cuves de chaque exploitation. Toutes les caractéristiques mesurées sur les laits individuels l'ont également été sur les laits de mélange pour lesquels, en plus, la teneur en caséines a été déterminée. Des mesures rhéologiques ont par ailleurs été effectuées environ 2 heures après la traite, sur des laits du matin non refroidis, à l'aide d'un gélographe (appareil qui trace une courbe d'évolution de la viscosité du lait emprésuré). Ces courbes permettent d'estimer le temps de coagulation, la vitesse maximum de raffermissement (plus grande pente de la courbe) et la

Tableau 1. Composition chimique et valeur nutritive moyenne $\left(^{\star}\right)$ des fourrages distribués au cours de l'hiver.

\begin{tabular}{|c|c|c|c|c|c|c|}
\hline \multirow[b]{2}{*}{ Exploitations } & \multicolumn{3}{|c|}{ Groupe 1} & \multicolumn{3}{|c|}{ Groupe 2} \\
\hline & 1 & 2 & 5 & 3 & 4 & 6 \\
\hline Cellulose Brute (\% MS) & 32 & 37 & 35 & 32 & 32 & 33 \\
\hline Matière Azotée Totale (\% MS) & 11,1 & 10,0 & $\mathbf{9}, 6$ & 11,6 & 9,8 & 9,7 \\
\hline Phosphore (g/kgMS) & 2,6 & 2,3 & 2,5 & 3,2 & 2,7 & 2,6 \\
\hline Calcium (g/kgMS) & 9,6 & 10,1 & 7,8 & 9,4 & 9,7 & 9,6 \\
\hline UFL (/kgMS) & 0,66 & 0,63 & 0,62 & 0,71 & 0,70 & 0,64 \\
\hline PDIN (g/kgMS) & 69 & 63 & 59 & 72 & 61 & 60 \\
\hline PDIE (g/kgMS) & 74 & 71 & 69 & 76 & 70 & 69 \\
\hline
\end{tabular}

$\left({ }^{*}\right)$ Moyennes sur les trois séries de mesure pondérées par les quantités ingérées des différents types de fourrages. 
Tableau 2. Caractéristiques des laits de cuve.

\begin{tabular}{|l|c|c|c|c|c|}
\hline & Moyenne & \multicolumn{2}{|c|}{ Groupe V } & \multicolumn{2}{c|}{ Groupe S } \\
& générale & $\mathbf{M}^{*}$ & $\mathbf{E}^{*}$ & $\mathbf{M}^{*}$ & $\mathbf{E}^{*}$ \\
\hline Taux butyreux (g/kg) & 36,4 & 37,1 & 9,1 & 35,6 & 7,0 \\
Taux protéique (g/kg) & 31,8 & 31,6 & 5,0 & 32,0 & 2,2 \\
Caséine (g/kg) & 24,7 & 24,9 & 4,1 & 25,1 & $\mathbf{1}, 8$ \\
Leucocytes (× 1000/ml) & 184 & 192 & 497 & 175 & 280 \\
Calcium (g/l) & 1,20 & 1,22 & 0,20 & $\mathbf{1 , 1 9}$ & $\mathbf{0 , 1 2}$ \\
Urée (mg/l) & 314 & 315 & 226 & 313 & $\mathbf{1 8 2}$ \\
pH & 6,73 & 6,73 & 0,25 & $\mathbf{6 , 7 3}$ & $\mathbf{0 , 1 1}$ \\
Acidité (" Dornic) & 18,2 & 18,4 & 4,2 & $\mathbf{1 7 , 9}$ & 3,0 \\
Indice de coagulation & 3,01 & 2,86 & 1,40 & 3,17 & $\mathbf{0 , 8 7}$ \\
Vitesse de raffermissement (mm) & 8,2 & $\mathbf{8 , 5}$ & 4,2 & $\mathbf{7 , 8}$ & 2,5 \\
Fermeté du gel * $2 \times$ temps de prise (mm) & 16,5 & 16,3 & 7,3 & $\mathbf{1 6 , 6}$ & 5,5 \\
Fermeté du gel ** 30 min (mm) & 16,2 & 14,3 & 14,0 & $\mathbf{1 8 , 2}$ & 11,9 \\
\hline
\end{tabular}

* $\mathrm{M}$ : moyenne entre janvier et avril 1989 ; $\mathrm{E}$ : écart extrême entre janvier et avril 1989.

** Les valeurs les plus faibles correspondent aux coagulums les plus fermes.

fermeté du gel 30 minutes après l'emprésurage ainsi qu'à deux fois le temps de coagulation.

\section{2 / Analyse des données}

L'analyse des données a été réalisée à plusieurs niveaux : après avoir décrit les moyennes et les évolutions des variables caractérisant les laits des cuves et l'alimentation des troupeaux, les caractéristiques du lait et de l'alimentation à l'échelle individuelle ont été analysées en janvier (mesure la plus proche du début de la lactation), en avril (juste avant la mise à l'herbe) et en juin (en alpage: les variables concernant l'alimentation des animaux n'étaient alors plus disponibles). Les données individuelles, qui permettent de confirmer et de préciser les phénomènes observés sur les laits des cuves ont été décrites par analyse factorielle des correspondances (AFC, Logiciel STAT-ITCF) (cf annexe). Seules les variables caractérisant la composition physico-chimique des laits individuels ont pris part à la construction des axes principaux; les autres variables disponibles (alimentation, poids, âge) ont été introduites comme variables supplémentaires. Les résultats étant identiques lors de chaque période étudiée, seules les analyses effectuées en avril seront présentées.

\section{2 / Résultats}

\section{1 / Caractéristiques des laits de cuve}

En moyenne, les taux butyreux et protéiques ont été respectivement de 36,4 et $31,8 \mathrm{~g} / \mathrm{kg}$ pour une production journalière de $17,3 \mathrm{~kg} /$ vache. Le taux butyreux a augmenté fortement lors de la mise à l'herbe $(+5,0 \mathrm{~g} / \mathrm{kg}$ entre avril et juin) contrairement au taux protéique qui a été beaucoup plus stable au cours de la période de mesure (figure 1). Le nombre de cellules a été plus élevé pendant la période estivale (230000/ml contre $180000 / \mathrm{ml}$ en hiver) comme cela a déjà été observé par ailleurs
(Coulon et Lilas 1988 ; Coulon et al 1988). Si l'indice de coagulation n'a pratiquement pas varié au cours de l'année (4\% d'écart entre les périodes hivernale et estivale), le raffermissement du caillé a été plus rapide durant la période estivale (de $14 \%$ ) et le gel obtenu, $30 \mathrm{mn}$ après l'emprésurage et à deux fois le temps de prise, plus ferme (respectivement de 19 et $12 \%$ ) (figure 1). Globalement, les laits des cuves ont donc présenté une aptitude à la coagulation légèrement meilleure durant la période estivale.

Ces valeurs moyennes cachent des écarts inter-groupes et inter-troupeaux variables (tableau 2) : le lait des exploitations du groupe $\mathrm{S}$ a été caractérisé par un taux butyreux, un taux de calcium, un degré d'acidité et un rapport $\mathrm{Ca} / \mathrm{N}$ légèrement plus faibles que ceux du groupe V. Par contre, le pH et le taux d'urée ont été semblables et les différences de teneurs en protéines, en caséines et en cellules du lait ont été dûes uniquement à des valeurs très différentes enregistrées dans une exploitation (tableau 2). De même, les différences d'aptitude à la coagulation moyenne entre les 2 groupes ont été faibles (à l'avantage du groupe V) (tableau 2). Par contre, la variabilité d'une quinzaine à l'autre de ces différentes caractéristiques a toujours été plus élevée dans les exploitations du groupe $\mathrm{V}$ (figure 1), que ce soit pour la composition chimique du lait (l'écart entre les valeurs bi-mensuelles extrêmes du taux protéique a été de $5,0 \mathrm{~g} / \mathrm{kg}$ dans les exploitations du groupe $\mathrm{V}$ contre 2,2 dans celles du groupe S), la numération cellulaire (497000 contre 280000 cellules $/ \mathrm{ml}$ ), ou les variables d'aptitude à la coagulation des laits (pour l'indice de coagulation, l'écart entre ces valeurs extrêmes a été de 1,40 dans le groupe $\mathrm{V}$ contre 0,87 dans le groupe $S$ ) (tableau 2).

\section{2 / Quantités ingérées et bilans alimentaires}

Les animaux des 6 exploitations ont ingéré en moyenne sur les trois séries de mesure
Les laits présentent une meilleure aptitude à la coagulation en période estivale : raffermissement du caillé plus rapide et gel plus ferme. 
Figure 1. Evolution des caractéristiques des laits de cuve dans les deux groupes d'exploitations entre janvier et juillet 1989.

(La mise à l'herbe a lieu de fin avril à fin mai selon les exploitations).
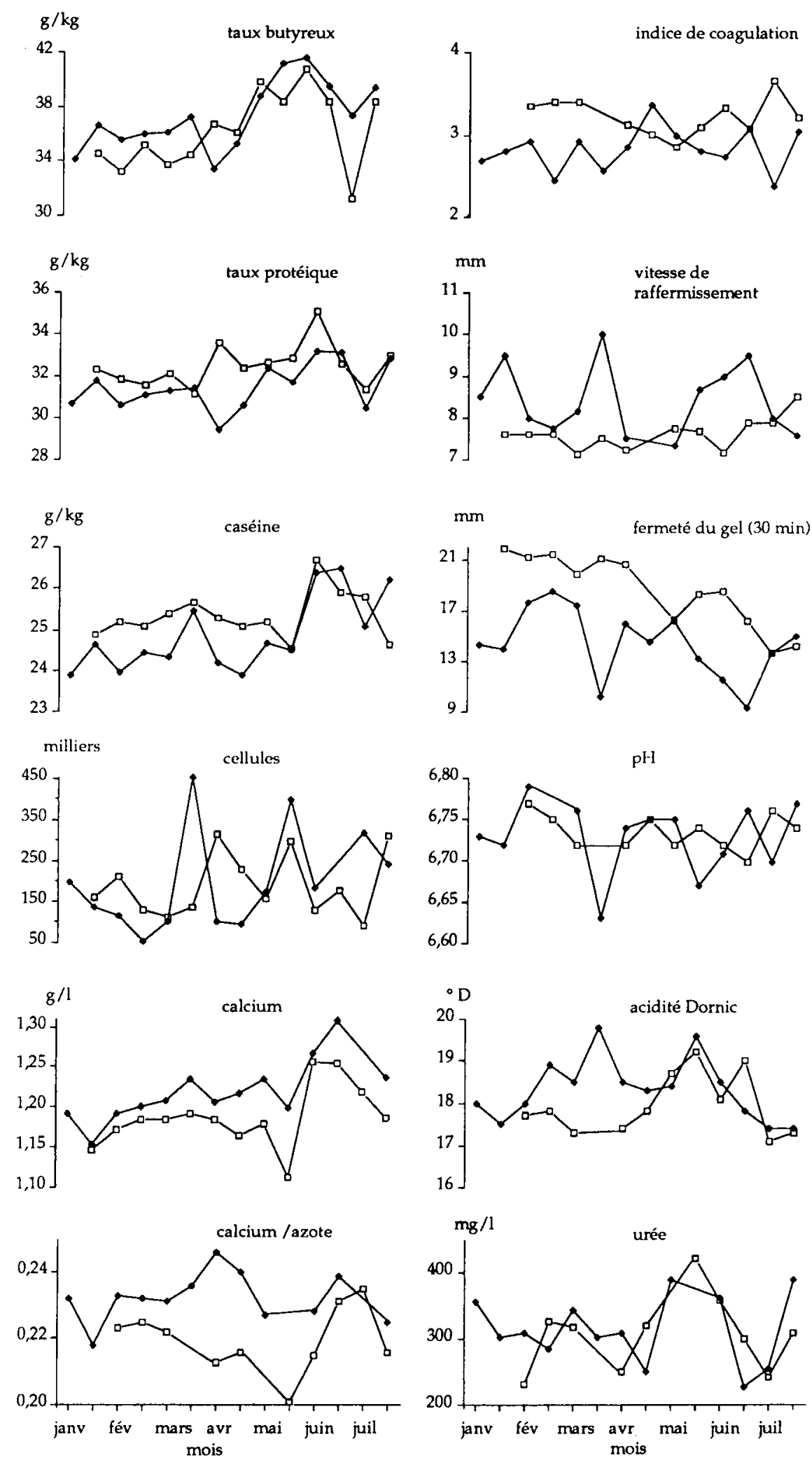

$12,0 \mathrm{~kg} \mathrm{MS} / \mathrm{j}$ de foin et 4,8 kg MS/j d'aliments concentrés. Compte tenu de la production laitière qui a varié de $17,9 \mathrm{~kg} /$ vache $/ \mathrm{j}$ en janvier à $16,7 \mathrm{~kg} / \mathrm{vache} / \mathrm{j}$ en avril, les bilans alimentaires ont été largement positifs (tableau 3) : l'excédent journalier en énergie, en azote, en phosphore et en calcium a été respectivement de 1,2 UFL (soit $10 \%$ des besoins), $217 \mathrm{~g}$ de PDI (soit $19 \%$ des besoins), $16 \mathrm{~g}$ de phosphore (30\% des besoins) et $89 \mathrm{~g}$ de calcium (94\% des besoins). Ces excédents ont été associés à des déséquilibres au niveau de l'alimentation azotée (excédentaire en PDIE, l'écart moyen PDIE-PDIN a été de 9,6 g PDI/UFL) et minérale (excédentaire en calcium, le rapport $\mathrm{Ca} / \mathrm{P}$ des apports étant de 2.61

Ces valeurs moyennes cachent de grosses différences inter-groupes (tableau 3): bien que dans les exploitations du groupe $\mathrm{V}$ le stade moyen de lactation ait été moins avancé (1 mois), la production individuelle inférieure de $3,4 \mathrm{~kg} / \mathrm{j}$, et les animaux de plus petit format, les quantités ingérées de fourrage et de concentré ont été pratiquement égales dans les 2 groupes d'exploitation. Les animaux des exploitations du groupe $V$ ont donc présenté des bilans énergétiques et azotés largement positifs, quelle que soit la période de mesure : + 1,9 UFL/j et +360 $\mathrm{g} \mathrm{PDI} / \mathrm{j}$ soit respectivement +18 et $+34 \%$ par rapport aux besoins. Dans les exploitations du groupe S, ces bilans ont également été positifs mais l'excédent n'a représenté en moyenne que 5 et $9 \%$ des besoins, respectivement pour l'énergie et l'azote. Ces résultats ont été peu variables à l'intérieur d'un même groupe, mais assez différents d'un mois à l'autre dans le groupe $S$, en liaison avec l'évolution du stade moyen de lactation (tableau 3).

En définitive, les exploitations du groupe V, par leurs besoins faibles et leurs bilans très excédentaires s'opposent aux exploitations du groupe $\mathrm{S}$ dans lesquelles la production individuelle est plus élevée et l'alimentation des animaux plus équilibrée malgré des variations hivernales importantes.

\section{3 / Liaison entre l'aptitude fromagère du lait et les bilans nutritifs}

A l'échelle des troupeaux, les bilans énergétiques, azotés ou minéraux ne permettent pas d'expliquer les variations d'aptitude à la coagulation des laits des cuves (figure 2), tout au moins dans la plage de variation des bilans disponibles (les bilans négatifs sont très peu nombreux]. Dans les conditions de bilans positifs, il semblerait que les plus forts excédents soient à mettre en relation avec les aptitudes à la coagulation les plus favorables. Cependant, lorsque l'on se place intra-exploitation, bien que les bilans énergétiques aient pu varier de -1 à +2 UFL/VL/jour (cas du troupeau 3), l'aptitude à la coagulation des laits de cuve est restée constante. Globalement, cette aptitude a surtout été liée à l'exploitation (figure 2). L'étude à l'échelle du troupeau a cependant été limitée par le faible nombre de données qui ne permet pas de s'affranchir de l'effet de l'exploitation et du stade physiologique des animaux. 
Tableau 3. Caractéristiques des animaux de chaque groupe d'exploitations et quantités ingérées au cours des 3 périodes de mesure.

\begin{tabular}{|c|c|c|c|c|c|c|c|c|c|}
\hline \multirow[b]{2}{*}{ Période } & \multirow[b]{2}{*}{$\begin{array}{c}\text { Poids } \\
\text { kg }\end{array}$} & \multirow{2}{*}{$\begin{array}{c}\text { Mois } \\
\text { de } \\
\text { lacta- } \\
\text { tion }\end{array}$} & \multirow[b]{2}{*}{$\begin{array}{l}\text { Lait } \\
\mathrm{kg} / \mathrm{j}\end{array}$} & \multirow{2}{*}{\multicolumn{2}{|c|}{$\begin{array}{c}\text { Quantités } \\
\text { ingérées } \\
\text { Foin Concentré } \\
\text { kgMS/j }\end{array}$}} & \multicolumn{4}{|c|}{ Bilans } \\
\hline & & & & & & $\underset{/ j}{\text { UFL }}$ & $\begin{array}{c}\text { PDI } \\
\mathrm{g} / \mathbf{j}\end{array}$ & $\underset{\mathrm{g} / \mathrm{j}}{\mathbf{P}}$ & $\begin{array}{l}\mathrm{Ca} \\
\mathrm{g} / \mathrm{j}\end{array}$ \\
\hline Janvier & 579 & 3,8 & 17,9 & 12,1 & 4,4 & 0,7 & 137 & 10 & 89 \\
\hline Mars & 577 & 4,8 & 17,2 & 11,9 & 5,3 & 1,7 & 352 & 21 & 94 \\
\hline Avril & 574 & 5,3 & 16,7 & 12,0 & 4,8 & 1,2 & 162 & 17 & 82 \\
\hline Moyenne générale & 577 & 4,6 & 17,3 & 12,0 & 4,8 & 1,2 & 217 & 16 & 89 \\
\hline Moyenne groupe V & 527 & 4,1 & 15,3 & 12,0 & 5,0 & 1,9 & 358 & 20 & 95 \\
\hline Moyenne groupe S & 612 & 5,0 & 18,7 & 12,1 & 4,7 & 0,7 & 116 & 13 & 85 \\
\hline
\end{tabular}

\section{4 / Liaisons entre les laits de cuve et les laits individuels}

\section{a / Effet du nombre de cellules et des variants génétiques des lacto-protéines}

Dans une des exploitations, du fait de la taille importante du troupeau, le lait était travaillé dans deux cuves séparées. Le lait d'une des cuves (petite cuve) a présenté une moins bonne aptitude à la coagulation que celui de l'autre cuve (l'indice de coagulation a été plus élevé (de $8 \%$ ), la vitesse maximum de raffermissement plus faible (de $26 \%$ ) et le gel obtenu $30 \mathrm{mn}$ après l'emprésurage moins ferme (de $46 \%$ )\} bien que le taux de matière utile, et en particulier de caséines ait été plus élevé que dans la grande cuve $[0,6 \mathrm{~g} / \mathrm{kg})$. D'autre part, le $\mathrm{pH}$, le taux d'urée, l'acidité du lait et le stade physiologique moyen des animaux ont été semblables dans les deux cuves (tableau 4) ; le lait de la petite cuve s'est différencié seulement par un nombre de leucocytes moyen nettement supérieur (336 000 contre 100000 cellules $/ \mathrm{ml}$ ) et un taux de Ca légèrement inférieur $(-0,04 \mathrm{~g} /$ 1).

(Un a unalumenl noté des différences de répartition des variants génétiques de la caséine kappa et de la B-lactoglobuline entre les deux cuves: les animaux de type BB pour la caséine kappa ont produit $31 \%$ de la matière protéique dans la grande cuve contre $7 \%$ dans la petite. Pour la béta-lactoglobuline, les animaux de type BB ont produit $23 \%$ des protéines de la grande cuve contre $8 \%$ dans la petite. Ces différentes répartitions des variants des lacto-protéines, en particulier du variant B de la caséine kappa, ont pu contribuer aux différences d'aptitude à la coagulation des laits des deux cuves (Grosclaude 1988, Aleandri et al 1990).

\section{b / Effet des caractéristiques des laits individuels}

Au mois d'avril, 156 vaches ont été contrôlées dont $28 \%$ de primipares. La majorité des animaux $(78 \%)$ étaient au delà de leur $3^{\prime \prime}$ mois de lactation. Les productions individuelles journalières ont été comprises entre 3 et $35 \mathrm{~kg}$. Le taux butyreux a élé environ deux fois plus
Figure 2. Liaisons entre les principales caractéristiques fromagères des laits de cuve et les bilans énergétiques moyens des troupeaux.

(Les mesures sur le lait et sur l'alimentation des animaux ont été effectuées à moins de 12 jours d'intervalle).
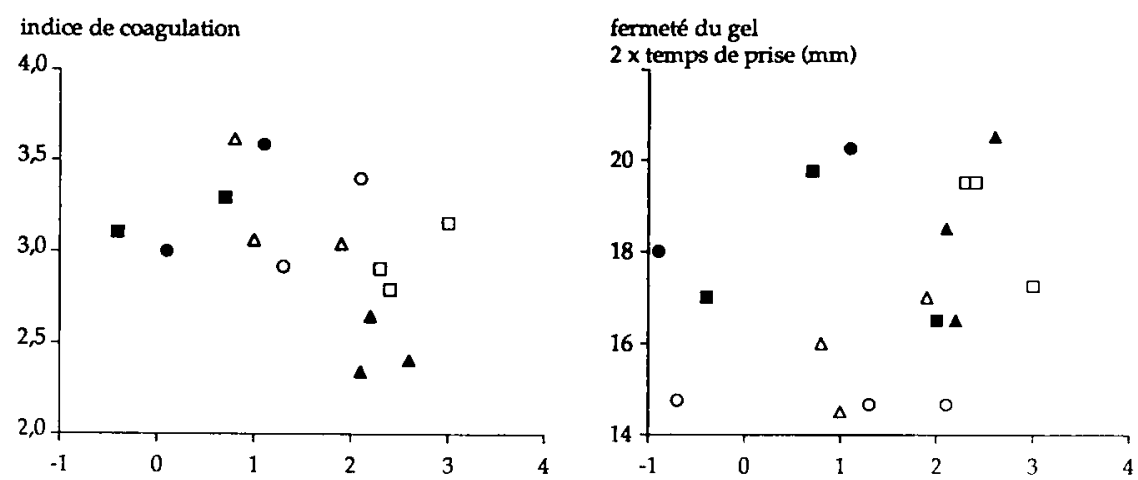

vitesse de raffermissement $(\mathrm{mm})$

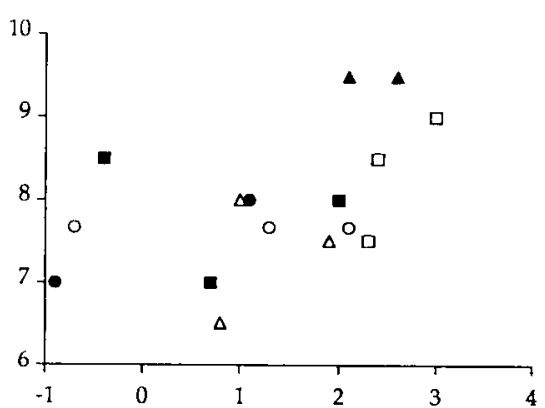

fermeté du gel $30 \mathrm{~min}(\mathrm{~mm})$

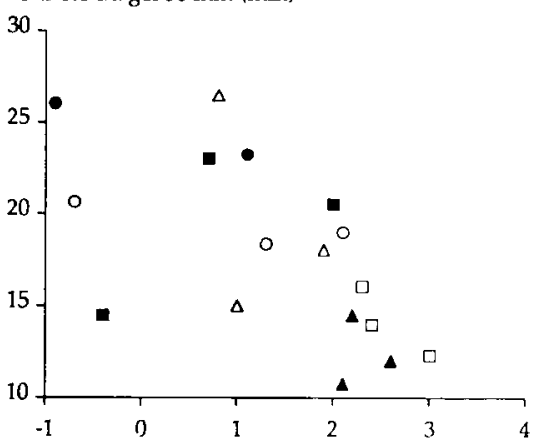

bilan énergétique (UFL / vache / jour)

variable que le taux protéique et $45 \%$ des animaux ont présenté des taux protéiques faibles $(<30 \mathrm{~g} / \mathrm{kg})$ et $/$ ou des taux butyreux très faibles $(<33 \mathrm{~g} / \mathrm{kg}$ ) (figure 3). L'indice de coagulation a varié du simple au quintuple, le $\mathrm{pH}$ de 6,6 à 6,9 et le taux d'urée de 200 à $500 \mathrm{mg} / \mathrm{l}$. Le nombre de leucocytes a été peu variable puisque $93 \%$ des laits ont présenté un nombre de leucocytes inférieur à $300000 / \mathrm{ml}$ et $2 \%$ seulement ont dépassé 1 million/ml. La majorité des animaux étaient en bilans énergétique et azoté positifs (respectivement 82 et $90 \%$ ). Ces bilans ont été compris entre $-3,9$ et $+5,0 \mathrm{UFL} / \mathrm{j}$ et entre $-290 \mathrm{et}+500 \mathrm{~g} \mathrm{PDI} / \mathrm{j}$. 
Tableau 4.

Caractéristiques moyennes des laits de cuves dans une exploitation. (moyenne entre janvier, mars et avril)

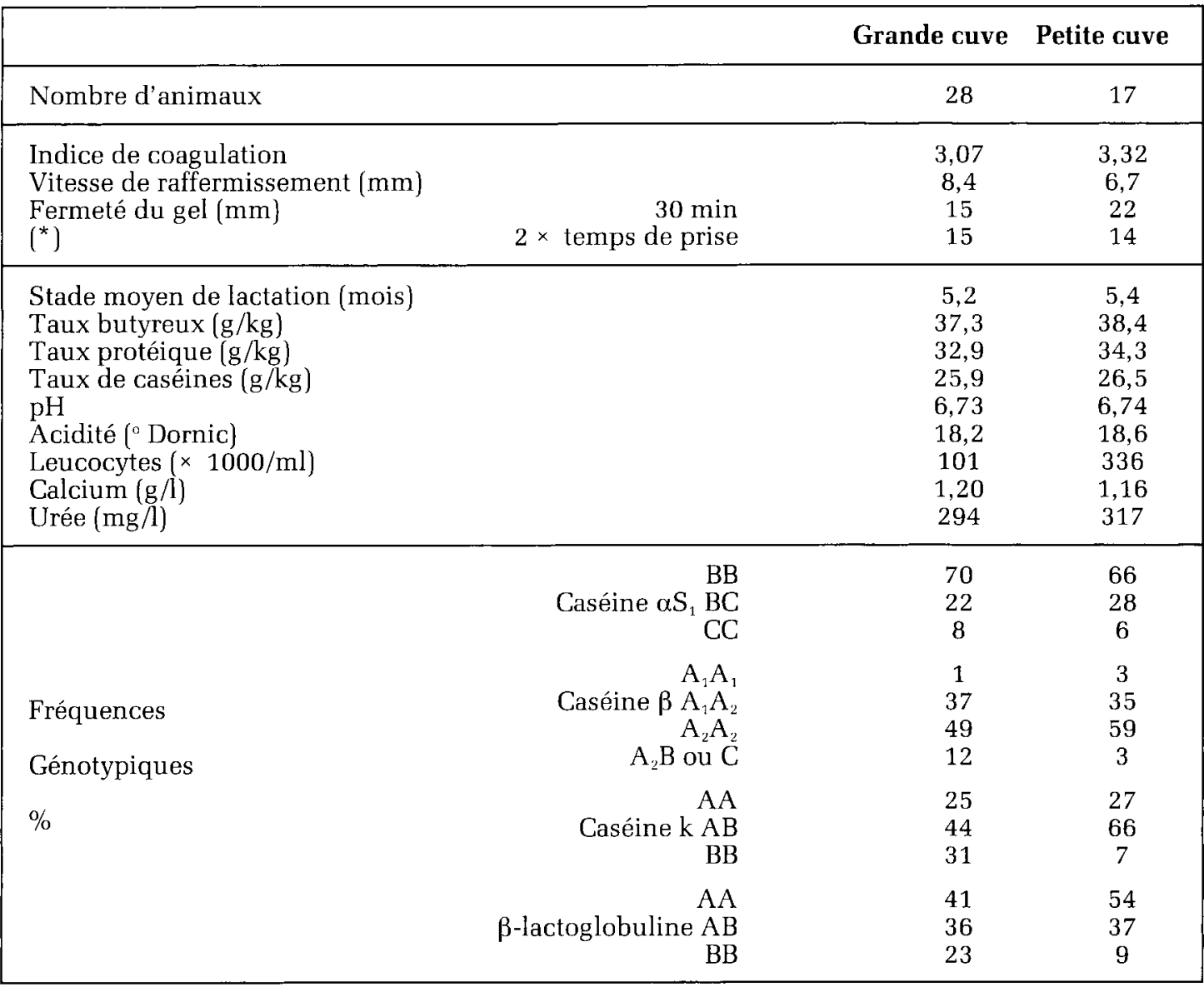

$\left({ }^{\star}\right)$ Les valeurs les plus faibles correspondent aux gels les plus fermes.

Figure 3. Variabilité individuelle des caractéristiques du lait (données du mois d'avril, $n=156$ ).

\section{nombre de vaches}
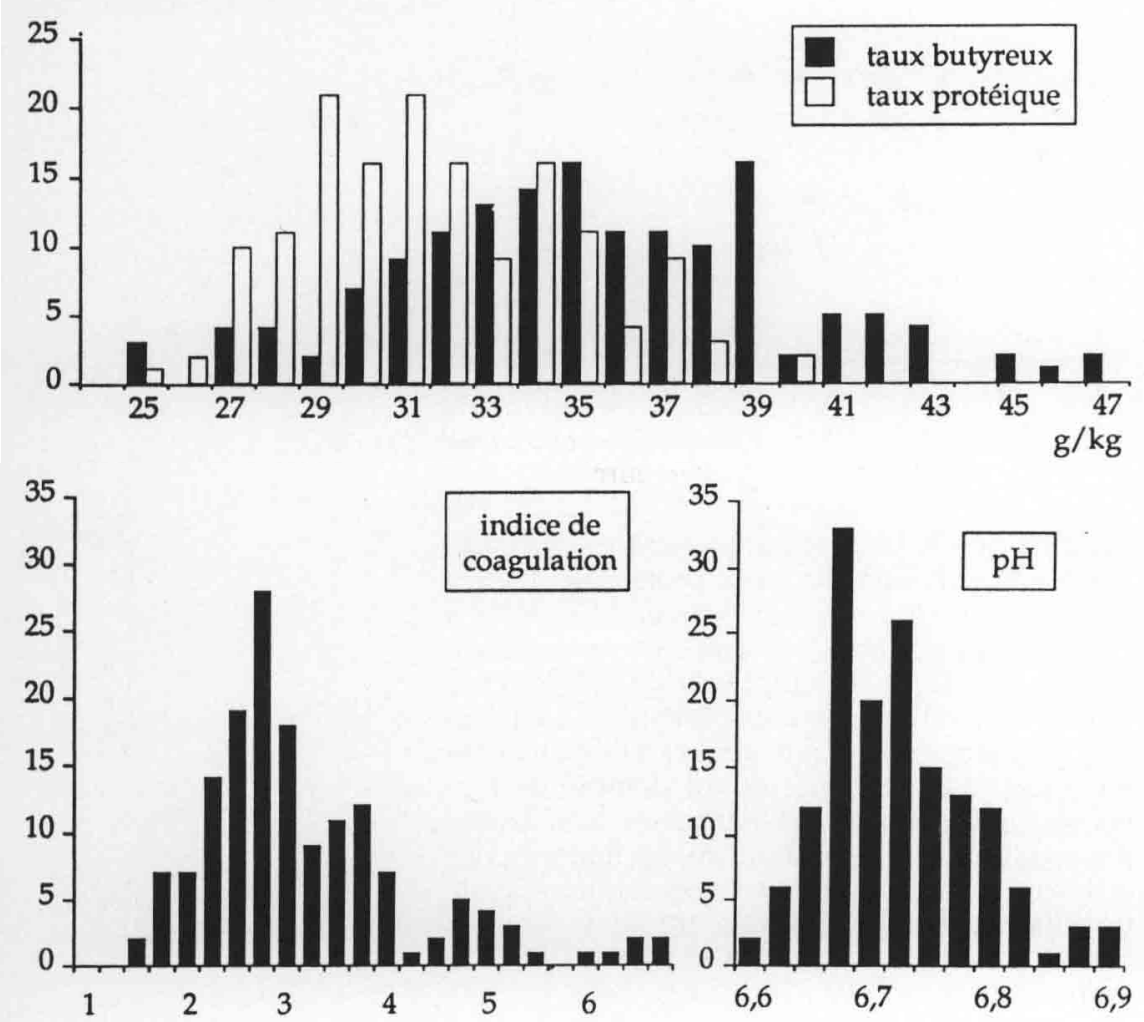

L'AFC (cf paragraphe 1.2) réalisée sur l'ensemble de ces animaux (figure 4) a permis de mettre en évidence 4 associations de facteurs discriminants pour l'indice de coagulation des laits. L'axe 1 oppose les laits de fin de lactation dont le taux protéique est élevé, aux laits de début de lactation plus pauvres en protéines. L'axe 2 permet d'opposer les laits en fonction de leur $\mathrm{pH}$ et de leur degré d'acidité Dornic. Les animaux produisant un lait qui coagule vite sont en début de lactation, leur lait est plutôt pauvre en protéines et est caractérisé par un $\mathrm{pH}$ faible.

Dans un deuxième temps, une AFC semblable a été réalisée sur les animaux en milieu de lactation uniquement, de manière à éliminer l'effet du stade physiologique des animaux sur l'indice de coagulation (Coulon et al 1991) L'étude du plan principal de cette AFC (figure 4) ne permet de mettre en évidence que deux associations de facteurs discriminants pour l'indice de coagulation: seul l'axe représentant le $\mathrm{pH}$ du lait permet de séparer les différentes modalités. Les variations de l'indice de coagulation apparaissent indépendantes de celles du taux protéique du lait.

Les deux analyses précédentes ne permettent pas de rapprocher les bilans alimentaires des animaux et l'indice de coagulation des laits: dans les deux cas, les différentes modalités des divers bilans calculés sont très mal représentées sur le plan principal et sont toutes regroupées autour de l'origine. Les bilans alimentaires des animaux et les critères de qualite lrumagere 
Figure 4. Représentation simplifiée du plan factoriel 1-2 des AFC réalisées sur les données relevées en avril 1989 .

Les variables mesurant lindice de coagulation (IC) sont reliées entre elles par ordre croissant de classes (cf annexe).

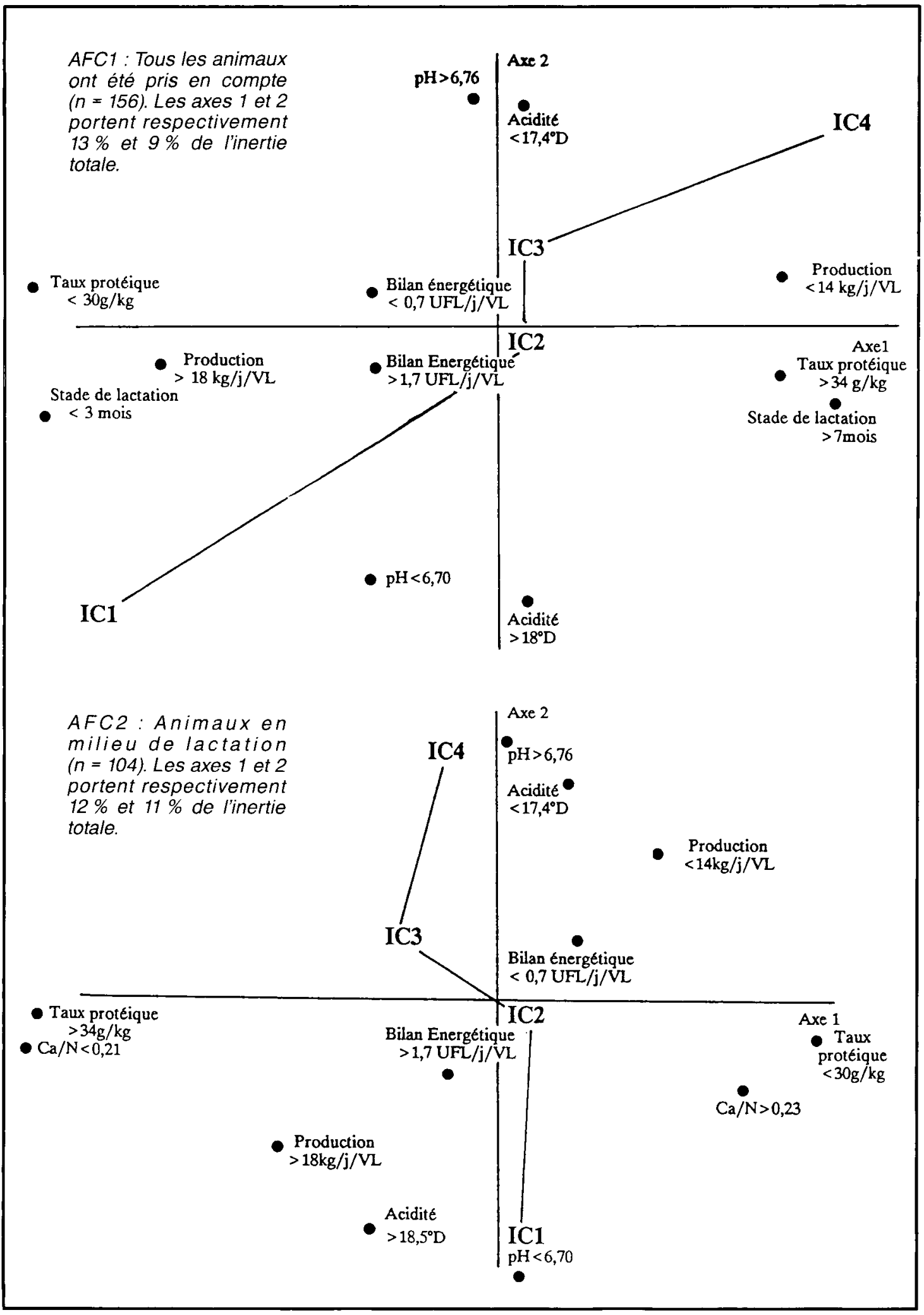

du lait semblent donc évoluer de façon indépendante. L'étude des corrélations entre les variables. sur la population entière (figure 5) ainsi que sul cles mpulations triées (intra stade physiologique et/ou intra exploitation), permet de confirmer l'absence de liaison entre le niveau d'alimentation des animaux et l'indice de coagulation de leur lait. Ces résultats confirment ainsi les observations réalisées à l'échelle du troupeau.
Les laits de début de lactation présentent un taux protéique et un $\mathrm{pH}$ plus faibles, et leur coagulation est plus rapide. Indépendamment du stade de lactation, l'indice de coagulation est essentiellement lié au $\mathrm{pH}$ du lait. 
Figure 5. Liaisons entre les bilans énergétiques, la teneur en urée du lait et lindice de coagulation des laits individuels (données du mois d'avril, $n=156)$.

indice de coagulation

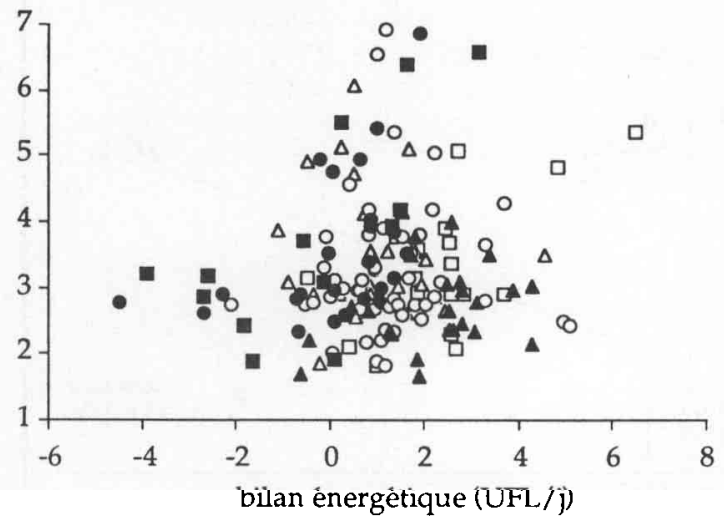

indice de coagulation

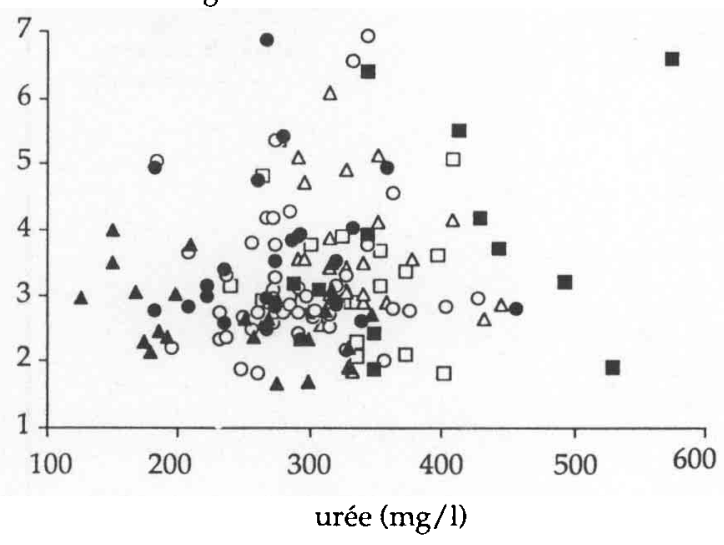

\section{c / Liaisons entre les différentes caractéristiques du lait}

L'étude des liaisons entre les critères individuels de qualité du lait pris deux à deux a permis de confirmer que l'indice de coagulation était d'autant plus faible que le $\mathrm{pH}$ du lait était plus bas $(r=0,57, P<0,01)$ (figure 5) et le rapport $\mathrm{Ca} / \mathrm{N}$ plus élevé $(\mathrm{r}=-0,31, \mathrm{P}<0,01)$. Par contre, l'indice de coagulation n'a été lié de façon significative, ni au taux d'urée, (figure 5) ni aux niveaux de production, ni au nombre de cellules du lait (dans la plage de variation observée). D'autre part, comme cela a déjà été observé par ailleurs (Delacroix 1985 ; Coulon et al 1988), le taux protéique a varié dans le même sens que l'acidité du lait $(\mathrm{r}=0,60, \mathrm{P}<0,01)$.

\section{Discussion - Conclusion}

Cette étude a mis en évidence les particularités des conduites alimentaires des troupeaux du Pays de Thônes où les apports énergétiques, azotés et minéraux ont été généralement largement supérieurs aux besoins des animaux. De tels niveaux d'alimentation ont déjà été observés dans les Alpes du Nord, vraisemblablement en raison du rapport très favorable: prix lait/ prix du concentré (Liénard et Baud 1981). Les forts excédents observés ont en effet été liés à une distribution importante d'aliments concentrés $(30 \%$ de la matière sèche totale, $40 \%$ des apports énergétiques) compte tenu du niveau de production des animaux et de la qualité des foins distribués. Ces forts niveaux d'alimentation hivernale peuvent expliquer que, dans la plupart des exploitations, les variations du taux protéique à la mise à l'herbe restent faibles contrairement à ce qui est souvent observé par ailleurs lorsque les niveaux alimentaires hivernaux sont plus faibles (Coulon et Binet 1987 ; Agabriel et al 1990).

La sur-alimentation hivernale, très marquée dans les trois exploitations où le niveau de production a été le plus faible (groupe V) ne s'est traduite ni par des quantités de lait produites plus importantes ni par des taux protéiques plus élevés que ceux des exploitations du groupe S. Cette absence d'effet est certainement dûe, en premier lieu, à des niveaux génétiques très différents d'un groupe à l'autre. Elle peut d'autre part illustrer la loi des rendements marginaux (Coulon et Rémond 1991) ; au delà de la couverture des besoins, la réponse de la production laitière à un apport alimentaire supplémentaire devient de plus en plus faible, au profit de la reconstitution des réserves corporelles, dont on ne disposait pas d'indicateur (note d'état d'engraissement) dans cette étude. Les forts déséquilibres enregistrés au niveau de l'alimentation azotée et minérale ont aussi pu se traduire par une moins bonne utilisation digestive de l'énergie ingérée. Elle doit enfin être interprétée avec prudence: il s'agit ici d'observations inter-exploitations sur un effectif très faible et non inter-individus sur une large population.

Les résultats obtenus à l'échelle du troupeau et de l'individu vont dans le même sens et mettent en évidence l'absence de liaison entre les bilans alimentaires des animaux (UFL, PDI, P et $\mathrm{Ca}$ ) et les paramètres de coagulation des laits (indice de coagulation mais aussi, vitesse de raffermissement ou fermeté du gel). Ils confirment ainsi les résultats obtenus dans des situations expérimentales et sur des plages de variation des bilans alimentaires différentes (Macheboeuf et Coulon, non publié). Ces observations vont également dans le même sens que celles de Vertès et al (1989) qui n'ont pas observé de liaison entre le niveau de l'alimentation hivernale et la phase primaire de la coagulation bien que la fermeté du gel ait été améliorée (parallèlement à une augmentation de la teneur en caséines du lait) par des apports alimentaires accrus, mais de nature différente.

En définitive, cette étude a confirmé l'existence de différences importantes d'une exploitation à l'autre des caractéristiques du lait et a permis de relever, dans certaines de celles-ci, de fortes variations de ces caractéristiques au cours de l'hiver. Elle a ainsi confirmé les résultats qualitatifs de l'enquête précédente concernant la plus grande variabilité des caractéristiques du lait des exploitations où la maîtrise du système d'élevage était médiocre, le niveau de production le plus faible et la valorisation du fromage la moins bonne. C'est cette variabilité que les producteurs fermiers ont du mal à gérer et qui se traduit sur les fromages par une dépré- 
ciation de la qualité et donc de la valorisation du lait (Coulon et al 1988). Les facteurs génétiques, qui jouent plus sur le niveau des variables que sur leur variabilité, ne sont donc a priori pas en cause, sauf s'ils agissent en interaction avec des facteurs du milieu. Cette étude montre que les bilans alimentaires ne permettent pas non plus à eux seuls d'expliquer les variations brutales de l'aptitude à la coagulation des laits de cuve. Cependant, dans cette étude, le choix des variables explicatives (concernant exclusivement le niveau des apports nutritifs), la fréquence des mesures (tous les 15 jours pour les laits de cuve mais seulement tous les mois pour les laits individuels, ce qui n'a pas permis de relier de manière fiable les variations de ces 2 sources d'information) et la finesse des observations n'ont pas permis d'appréhender des variables de conduite des animaux autres qu'alimentaires (mouvements d'animaux, traite, facteurs sanitaires ...) ou d'ordre plus qualitatif (nature de la ration, méthode d'apport et de transition, ...). D'autre part, seules des variables décrivant la coagulation du lait ont été étudiées. Afin d'essayer de préciser l'influence réelle des caractéristiques du système d'élevage sur les variations de l'aptitude fromagère du lait au cours de l'année (et en particulier sur la qualité des fromages affinés), des travaux spécifiques sont actuellement entrepris, en situation expérimentale et dans des exploitations des Alpes du Nord.

\section{Remerciements}

Nous tenons à remercier X. Folliet, le Contrôle Laitier de Haute-Savoie, le Syndicat Interprofessionnel du Reblochon (Y. Burnet-Merlin), la coopérative de Reblochon Fermier de Thônes et L'ITG pour leur participation aux prélèvements et mesures et leurs conseils

\section{Références bibliographiques}

AGABRIEL C., COULON J.B., MARTY G., CHENEAU N., 1990. Facteurs de variation du taux protéique du lait de vache. Etude dans les exploitations du Puy-de-Dôme. INRA, Prod. Anim., 1990, 3, 137-150.

ALEANDRI R., BUTTAZONI L.G., SCHNEIDER J.C. 1990. The effect of milk protein polymorphisms on milk components and cheese-producing ability. J. Dairy Sci. $73,241-255$.

COULON J.B., BINET M., 1987. Facteurs de variation du taux protéique du lait de vache en exploitation. Etude dans l'aire de ramassage de la coopérative fromagère agricole de Laguiole (Aveyron). Bull. Tech. CRZV Theix INRA, $68,11-18$.

COULON J.B., LILAS J.P., 1988. Composition chimique et contamination butyrique du lait: facteur de variation dans le département de la Haute-Loire. INRA Prod Anim., 1, 201-207.

COULON J.B., ROYBIN D., CONGY E.,GARRET A., 1988 Composition chimique et temps de coagulation du lait de vache : facteurs de variations dans les exploitations do Pays de Thônes. INRA Prod. Anim., 1, 253-263.

COULON J.B., REMOND B., 1991. Réponses de la production et de la composition du lait de vache aux variations d'apports nutritifs. INRA Prod. Anim., 4, 49-56.

COULON J.B., CHILLIARD Y., REMOND B., 1991. Effets du stade physiologique et de la saison sur la composition chimique du lait de vache et ses caractéristiques technologiques (aptitude à la coagulation, lipolyse). INRA Prod. Anim., 4 (3), 219-228.

DELACROIX A.. 1985. Ftude des variations individuelles de la composition chimique (fractions azotées et minérales) et de quelques caractéristiques des laits de vache en fonction de l'alimentation. Thèse de Docteur-Ingénieur ENSA Rennes.

GROSCLAUDE F., 1988. Le polynorphisme génétique des principales lactoprotéines bovines. Relations avec la quantité, la composition et les aptitudes fromagères du lait. INRA Prod. Anim., 1, 5-17.

LIENARD G, BAUD G., 1981. Les problèmes de la production laitière en montagne. In: La production laitière française - évolution récente et perspectives. Ed INRA Publ., route de St-Cyr. 78000 Versailles.

VERMOREL M., COULON J.B., JOURNET M., 1987. Révision du système des Unités Fourragères (UF). Bull. Tech. CRZV Theix, INRA, 70, 9-18.

VERTES C., HODEN A., 1989. Qualité fromagère des laits de vache en fonction des régimes à base d'herbe. Le Lait, 69, 197-209.

VERTES C., HODEN A. GALLARD Y., 1989. Effet du niveau d'alimentation sur la composition chimique et la qualité fromagère du lait de vaches Holstein et Normandes. Résultats préliminaires. INRA Prod. Anim., 2, $89-96$

\section{Summary}

Renneting properties of dairy cows milk: influence of nutritional level.

This study was carried out between January and July 1989, in 6 dairy farms making cheese (Reblochon), located in the Thônes area (Haute-Savoie). Coagulation properties (coagulation time, curd firming rate and curd firmness) were described and related to the feeding characteristics of the cows (energy, nitrogen and mineral balances) and with the chemical characteristics of the milk (fat and protein content, $\mathrm{pH}$, etc). In all the farms the nutritional balances were positive, more so in those where milk characteristics were the more variable throughout the year. Using herd values $(n=6)$ or individual values $(n=160)$, it was not possible to relate the variations in coagulation properties with those of nutritional balances of the cows. The variations in coagulation properties were well related with those of milk pH or with the physiological stage of the cows. Other feeding factors (feed type etc) or non-feeding factors (milking, health, genetic factors) may be responsible for these variations.

MARTIN B., COULON J.B.,1991. Aptitude à la coagulation du lait de vache : Influence de l'alimentation. Etude réalisée dans 6 exploitations du Pays de Thônes (Haute-Savoie). INRA Prod. Anim., 4 (3), 209-217. 
Annexe. Critères retenus dans les analyses factorielles des correspondances.

\begin{tabular}{|c|c|c|c|c|}
\hline \multicolumn{5}{|c|}{ CARACTÉRISTIQUES DU LAIT } \\
\hline \multirow[b]{2}{*}{ Critères } & \multicolumn{2}{|c|}{ Tous les animaux $(n=156)$} & \multicolumn{2}{|c|}{$\begin{array}{l}\text { Animaux en milieu } \\
\text { de lactation }(n=104)\end{array}$} \\
\hline & Classes & Effectif & Classes & Effectif \\
\hline $\begin{array}{l}\text { Production laitière } \\
\text { (kg/jour) }\end{array}$ & $\begin{array}{c}4,9-14,2 \\
14,2-19,2 \\
19,2-32,6\end{array}$ & $\begin{array}{l}52 \\
52 \\
52\end{array}$ & $\begin{array}{c}4,9-13,5 \\
13,5-18,5 \\
18,2-28,6\end{array}$ & $\begin{array}{l}35 \\
35 \\
34\end{array}$ \\
\hline Taux butyreux $(\mathrm{g} / \mathrm{kg})$ & $\begin{array}{l}20,0-33,6 \\
33,6-37,8 \\
37,8-48,0\end{array}$ & $\begin{array}{l}52 \\
52 \\
52\end{array}$ & $\begin{array}{c}25,6-33,7 \\
33,7-37,8 \\
37,8-47\end{array}$ & $\begin{array}{l}35 \\
35 \\
34\end{array}$ \\
\hline Taux protéique $(\mathrm{g} / \mathrm{kg})$ & $\begin{array}{l}20,0-30,0 \\
30,0-33,7 \\
33,7-40,0\end{array}$ & $\begin{array}{l}52 \\
52 \\
52\end{array}$ & $\begin{array}{l}20,0-30,0 \\
30,0-33,5 \\
33,5-38,7\end{array}$ & $\begin{array}{l}35 \\
35 \\
34\end{array}$ \\
\hline $\begin{array}{l}\text { Nombre de leucocytes } \\
\text { (milliers } / \mathrm{ml} \text { ) }\end{array}$ & $\begin{array}{c}0-100 \\
100-300 \\
300-2855\end{array}$ & $\begin{array}{l}108 \\
37 \\
11\end{array}$ & $\begin{array}{c}0-100 \\
100-300 \\
300-2855\end{array}$ & $\begin{array}{c}73 \\
23 \\
8\end{array}$ \\
\hline Taux de calcium $(\mathrm{g} / \mathrm{l})$ & $\begin{array}{l}0,99-1,15 \\
1,15-1,23 \\
1,23-1,58\end{array}$ & $\begin{array}{l}52 \\
52 \\
52\end{array}$ & $\begin{array}{l}1,00-1,15 \\
1,15-1,22 \\
1,22-1,43\end{array}$ & $\begin{array}{l}35 \\
35 \\
34\end{array}$ \\
\hline Taux d'urée (mg/l) & $\begin{array}{l}125-273 \\
273-327 \\
327-811\end{array}$ & $\begin{array}{l}55 \\
52 \\
49\end{array}$ & $\begin{array}{l}125-280 \\
280-320 \\
320-811\end{array}$ & $\begin{array}{l}35 \\
36 \\
33\end{array}$ \\
\hline $\mathrm{pH}$ & $\begin{array}{l}6,53-6,70 \\
6,70-6,76 \\
6,76-7,16\end{array}$ & $\begin{array}{l}54 \\
53 \\
49\end{array}$ & $\begin{array}{l}6,61-6,70 \\
6,70-6,75 \\
6,75-7,15\end{array}$ & $\begin{array}{l}33 \\
36 \\
35\end{array}$ \\
\hline Acidité ( ${ }^{\circ}$ Dornic $)$ & $\begin{array}{l}12,7-17,4 \\
17,4-18,5 \\
18,5-24,9\end{array}$ & $\begin{array}{l}53 \\
49 \\
54\end{array}$ & $\begin{array}{l}12,7-17,6 \\
17,6-18,5 \\
18,5-21,1\end{array}$ & $\begin{array}{l}35 \\
34 \\
35\end{array}$ \\
\hline Rapport $\mathrm{Ca} / \mathrm{N}$ & $\begin{array}{l}0,16-0,21 \\
0,21-0,24 \\
0,24-0,35\end{array}$ & $\begin{array}{l}52 \\
52 \\
52\end{array}$ & $\begin{array}{l}0,17-0,21 \\
0,21-0,24 \\
0,24-0,35\end{array}$ & $\begin{array}{l}35 \\
35 \\
34\end{array}$ \\
\hline Indice de coagulation & $\begin{array}{l}1,6-2,6 \\
2,6-3,0 \\
3,0-3,8 \\
3,8-6,9\end{array}$ & $\begin{array}{l}39 \\
39 \\
38 \\
40\end{array}$ & $\begin{array}{l}1,9-2,7 \\
2,7-3,1 \\
3,1-3,9 \\
3,9-6,9\end{array}$ & $\begin{array}{l}26 \\
27 \\
26 \\
25\end{array}$ \\
\hline \multicolumn{5}{|c|}{ CAR ACTÉRISTIQUES DES ANIMAUX } \\
\hline Poids (kg) & $\begin{array}{l}450-520 \\
520-600 \\
600-750\end{array}$ & $\begin{array}{l}50 \\
56 \\
50\end{array}$ & $\begin{array}{l}450-520 \\
520-600 \\
600-750\end{array}$ & $\begin{array}{l}35 \\
36 \\
33\end{array}$ \\
\hline Stade de lactation (mois) & $\begin{array}{c}1-3 \\
4-7 \\
8-10\end{array}$ & $\begin{array}{c}34 \\
104 \\
18\end{array}$ & $4-7$ & 104 \\
\hline Numéro de lactation & $\begin{array}{c}1 \\
2-3 \\
4-12\end{array}$ & $\begin{array}{l}43 \\
63 \\
50\end{array}$ & $\begin{array}{c}1 \\
2-3 \\
4-12\end{array}$ & $\begin{array}{l}36 \\
33 \\
35\end{array}$ \\
\hline \multicolumn{5}{|c|}{ ALIMENTATION DES ANIMAUX } \\
\hline $\begin{array}{l}\text { Aliments concentrés } \\
(\mathrm{kg} \text { brut } / \mathrm{j})\end{array}$ & $\begin{array}{c}0-4,5 \\
4,5-6,3 \\
6,3-9,0\end{array}$ & $\begin{array}{l}52 \\
60 \\
44\end{array}$ & $\begin{array}{l}2,6-4,6 \\
4,6-6,2 \\
6,2-9,0\end{array}$ & $\begin{array}{l}36 \\
25 \\
43\end{array}$ \\
\hline $\begin{array}{l}\text { Aliments minéraux } \\
(\mathrm{g} / \mathrm{j})\end{array}$ & $\begin{array}{c}55-85 \\
85-96 \\
96-130\end{array}$ & $\begin{array}{l}68 \\
51 \\
37\end{array}$ & $\begin{array}{c}55-85 \\
85-96 \\
96-130\end{array}$ & $\begin{array}{l}45 \\
36 \\
23\end{array}$ \\
\hline $\begin{array}{l}\text { Bilans énergétiques } \\
\text { (UFL/jour) }\end{array}$ & $\begin{array}{l}-4,7-+0,7 \\
+0,7-+1,7 \\
+1,7-+6,5\end{array}$ & $\begin{array}{l}52 \\
52 \\
52\end{array}$ & $\begin{array}{l}-2,7-+0,7 \\
+0,7-+1,8 \\
+1,8-+6,5\end{array}$ & $\begin{array}{l}35 \\
35 \\
34\end{array}$ \\
\hline Bilans PDIE (g/jour) & $\begin{array}{l}-315-+244 \\
+244-+357 \\
+357-+993\end{array}$ & $\begin{array}{l}52 \\
52 \\
52\end{array}$ & $\begin{aligned} & -80-+250 \\
+ & 250-+350 \\
+ & 350-+992\end{aligned}$ & $\begin{array}{l}35 \\
35 \\
34\end{array}$ \\
\hline Bilans PDIN (g/jour) & $\begin{array}{c}-423-+60 \\
+60-+191 \\
+191-+992\end{array}$ & $\begin{array}{l}52 \\
52 \\
52\end{array}$ & $\begin{array}{c}-227+70 \\
+70-+191 \\
+191-+944\end{array}$ & $\begin{array}{l}35 \\
35 \\
34\end{array}$ \\
\hline $\begin{array}{l}\text { Bilans Phosphore } \\
\text { (g/jour) }\end{array}$ & $\begin{array}{l}-10-+14 \\
+14-+19 \\
+19-+40\end{array}$ & $\begin{array}{l}52 \\
52 \\
52\end{array}$ & $\begin{array}{l}-2-+14 \\
+14-+19 \\
+19-+40\end{array}$ & $\begin{array}{l}35 \\
35 \\
34\end{array}$ \\
\hline Bilans Calcium (g/jour) & $\begin{array}{l}+20-+80 \\
+80-+87 \\
+87-+138\end{array}$ & $\begin{array}{l}52 \\
52 \\
52\end{array}$ & $\begin{array}{l}+32-+80 \\
+80-+87 \\
+87-+137\end{array}$ & $\begin{array}{l}35 \\
35 \\
34\end{array}$ \\
\hline
\end{tabular}

